\title{
LA FUTAIE PAR BOUQUETS *
}

Les ouvrages techniques du $\mathrm{XIX}^{\mathrm{e}}$ siècle méritent d'être relus et médités. Ils représentent un " plantureux humus d'idées ) dont nos devanciers ont certes tiré parti, mais sans en épuiser les possibilités.

La "futaie par bouquets " nous en fournit un bel exemple: imaginée et exposée dans son principe dès I880, elle n'a reçu de véritable application qu'après I920. De même que le jardinage et le contrôle ont demandé de longs cheminements pour être mis au point à partir des idées de GurNaud, de même une technique aussi " affinée ) que la localisation des espèces forestières par nature de station exigeait des précisions, fournies aujourd'hui par la Pédologie et la Phytosociologie.

Nous nous proposorıs d'étudier d'abord les circonstances dans lesquelles a été conçue cette notion de futaie par bouquets et de préciser les buts auxquels elle répondait. Nous en verrons ensuite le perfectionnement jusqu'à la technique actuelle et les difficultés qu'on rencontre à la concilier avec certaines doctrines d'aménagement. Enfin, nous chercherons à indiquer les cas particuliers ou généraux auxquels cette technique pourrait être appliquée.

La Sylviculture du $\mathrm{XIX}^{\mathrm{e}}$ siècle a procédé par masses beaucoup plus que par détails. Elle a harmonisé les tentatives antérieures de remise en ordre des forêts après des siècles de récolte déréglée. Elle a pris conscience des lois présidant à la reconstitution des peuplements exploités et des possibilités d'échelonnement dans le temps d'opérations dirigées. Elle a codifié les traitements à appliquer et les a vus simples.

Pour reproduire cette schématisation sur le terrain, le forestier a dû violenter quelque peu la nature, qui est généralement complexe, qui est toujours prodigue, et qui répète rarement les mêmes phénomènes avec la fixité désirée par l'homme. Contre la complexité, le forestier a lutté en simplifiant, et il a pris le goût de l'homogénéité. Contre la prodigalité, il a réagi en économisant et il a cherché la rentabilité. Contre la variabilité, il a manifesté son autorité en en supprimant les causes, et il a voulu la pureté, au besoin en créant artificiellement le peuplement jugé idéal.

(*) Conférence donnée à Nancy à l'occasion de la Semaine d'information (21-26 avril 1952). Rev. forest, franç., mai 1952, p. 362 , 
Le résultat de ces tendances s'est manifesté dans les aménagements de peuplements mis au point au cours du siècle dernier et qui modèlent les forêts en grandes masses homogènes.

$\mathrm{Si}$, en France, les aménagements par affectations, issus de la méthode du réensemencement naturel et des éclaircies. restent basés sur l'éducation de peuplements dans la création desquels interviennent au maximum les forces naturelles, il n'en reste pas moins que cette éducation est faite au profit d'une seule essence productive, ici le chêne, là le hêtre, ailleurs le pin sylvestre ou le sapin.

Compréhensible dans le cas de futaies de plaine créées depuis plus d'un siècle, cette recherche sur le grandes surfaces de peuplements homogènes apparaît moins logique dans le traitement des taillissous-futaie en transformation. Le forestier dispose, en effet, d'éléments disparates qu'il peut faire évoluer à sa guise; il bénéficie en particulier, dans la réserve, d'une richesse en espèces que présentent rarement les peuplements pleins. Et, cependant, les aménagements de conversion par la méthode classique auront pour effet, et peut-être pour but (?), de mener ces anciens taillis-sous-futaie vers une forme homogène dont se trouveront peu à peu éliminées les essences autres que celles pour lesquelles l'aménagement a été réglé.

Nous verrons plus loin quelles difficultés peuvent naitre de l'application systématique de ces méthodes, lorsque les opérations de régénération (ou de conversion) relativement faciles dans les premières affectations, doivent être poursuivies dans les parcelles rejetées en fin de révolution et qui s'avèrent trop différentes des premières pour suivre le même sort.

Ainsi se manifeste, même en plaine, une évolution des peuplements qui déjoue les prévisions de l'aménagiste. A plus forte raison, le traitement des forêts de basse ou moyenne montagne ne peutil s'inscrire dans un cadre trop rigide. Et la mise en exploitation réglée des forêts de montagne, à partir du milieu du XIX ${ }^{\bullet}$ siècle, se trouve à l'origine de méthodes assouplies qui, par contre-coup feront éclater les affectations de leur conception trop étroite.

L'évolution des idées se précise autour des années I88a avec les maitres que furent BAGnéris et BroIlliard. Sans doute restentils fidèles aux doctrines de Lorentz et PARADE au moins dans leurs écrits ; mais leur enseignement sur le terrain est déjà plus nuancé. Ils constatent la supériorité de la " méthode d'observation qui constate les phénomènes, les compare, les étudie et en recherche les causes... ). Ils l'appliquent, eux et leurs élèves, " ...tout naturellement aux forêts, associations vivantes de végétaux, comme les bons conducteurs des peuples l'appliquent aux foules qui s'agitent )"(I).

Ils s'évadent quelque peu, de la méthode classique: Broilliard rompt l'uniformité en proposant l'éclaircie par le haut qui établit une hiérarchie entre les essences productives et les essences utiles au point de vue cultural; BAgNérIS, dès 1873 , consacre un chapitre de 
son “ Manuel de sylviculture ») (2) à la futaie jardinée qui n'était pas jusque-là reconnue comme mode de traitement. Et ils eussent sans doute été plus loin dans l'exposé de leurs tendances, s'ils n'avaient été retenus par un sentiment de réserve louable que justifie Broilliard quelques années plus tard: " Rendu à la liberté après avoir quitté l'administration, je n'avais plus à craindre cette fois, comme c'eût été possible en I86o, d'engager l'enseignement de l'Ecole, en émettant mes idées au grand large ) (I).

Sans doute, dans cette croisade contre l'excès de régularité, peuton relever des préoccupations idéologiques: par exemple GURNAUD apparait, à son époque, plus doctrinaire que chef de file. Il n'en reste pas moins que ce fut par nécessité et après les échecs de la méthode naturelle que les forestiers se tournèrent vers le jardinage d'abord, puis étendirent aux futaies pleines, quelques-uns des progrès que cette technique leur avait permis de réaliser.

En Allemagne, les conversions directes par substitution d'une essence résineuse aux taillis insuffisamment productifs, l'application fréquente, dans les futaies, de la coupe rase suivie de plantation, conduisent à des peuplements purs et homogènes. Les aménagements par compartiments qui leur sont appliqués, satisfont provisoirement l'esprit, mais se trouvent déjoués par l'évolution consécutive du peuplement et $\mathrm{du}$ sol. Les économistes les critiquent et obtiendront leur assouplissement.

Les sylviculteurs s'inquiètent des répercussions à plus ou moins longue échéance de ces traitements: "Nous sommes trop habitués à rechercher avant tout le produit et à négliger les agents producteurs; nous continuons à escompter les rendements d'autrefois, et nous ne savons pas reconnaitre la dilapidation de notre capital ni la nécessité de le sauvegarder ) (3, p. IX).

Cette phrase se trouve dans la préface de la $\mathrm{I}^{\mathrm{re}}$ édition du “ Traité de Sylviculture » de K. GAYER, parı à Münich en I880. L'auteur, après avoir souligné la rareté des peuplements mélangés et dénoncé le danger des monocultures, demande à ses contemporains « de rompre avec des traditions évidemment dangereuses et de chercher la vérité dans les leçons de la nature qui, seule, peut nous donner des indications infaillibles »).

Ainsi, en Allemagne comme en France, certains forestiers ne se déclarent pas satisfaits par la contemplation des futaies pleines, où sur de vastes étendues domine largement une seule essence productive.

Pour rompre l'uniformité et rétablir le mélange, GAYER propose la méthode de " futaie par bouquets ) souvent décrite sous le nom de “ Gayer'sche Femelschlagbetrieb ». La technique qu'il définit dans son Traité de Sylviculture revêt deux aspects principaux:

- ou bien, elle vise à amorcer la régénération en un certain nombre de points par l'ouverture de petites trouées élargies en taches 
d'huile jusqu'à se rejoindre. Le peuplement régénéré aura alors une forme vaguement ondulée qui s'atténuera par la suite.

- ou bien elle entr'ouvre des peuplements résineux purs et réguliers (pineraies, pessières de la plaine allemande), exposés par làmême aux catastrophes, pour introduire, dans les trouées ainsi faites et par plantation, des essences feuillues améliorantes qui faciliteront la régénération naturelle ultérieure de l'essence résineuse et assureront un mélange favorable dans le peuplement définitif.

Les applications qui furent faites de cette technique, dans la plupart des pays d'Europe jusqu'au premier quart de notre siècle, ne dépassèrent pas le stade de création des peuplements.

La méthode de régénération par trouées jardinatoires, par exemple, fait application de la première idée puisqu'elle tend à faire apparaître ou à dégager des groupes ou bouquets de semis naturels toutes les fois que les conditions naturelles rendent difficile ou aléatoire une régénération rapide et uniforme.

Cette technique sylvicole, fréquemment appliquée aux hêtraies ou aux sapinières, ne fait pas obstacle à l'application d'un aménagement en futaie régulière, car le vieillissement plus ou moins rapide des divers éléments aboutit souvent à une régularisation. "Le caractère qui résulte du mélange par bouquets se perd avec l'âge, quand l'étendue de ces bouquets formés dans la jeunesse n'a pas été exagérée » (3, p. 232).

Nous trouvons une remarquable application de la deuxième idée dans la méthode décrite par l'actuel Directeur Général des Forêts de Belgique, M. G. Turner, lorsqu'il expose les résultats de 25 ans d'expérimentations poursuivies dans la région de Vielsalm en vue de transformer les pessières pures en peuplements mélangés (4).

La forme la plus " affinée ) de la futaie par bouquets se trouve réalisée par le "Schweizerische Femelschlagbetrieb ) dont la technique est exposée et analysée plus loin en détail.

En parlant jusqu'ici de coupes jardinatoires, de régénération par bouquets ou par groupes, nous n'avons envisagé qu'une technique sylyicole à incorporer dans les "Règles de culture » avec d'autres méthodes de régénération ou d'entretien parmi lesquelles l'agent d'exécution est libre de choisir celle qui s'accorde le mieux avec le tempérament des essences, eu égard aux conditions de station.

Nous n'avons pas élevé la " futaie par bouquets ) au rang de méthode d'aménagement. Les traités classiques ne prévoient d'ailleurs que deux formes extrêmes à donner aux futaies qui doivent rentrer, soit dans le cadre des aménagements en futaies pleines, soit dans le cadre des aménagements en futaies jardinées.

HuFfeL reconnait bien que " ...les peuplements de nos sapinières sont presque partout dans un état qui n'est ni l'état équienne, au 


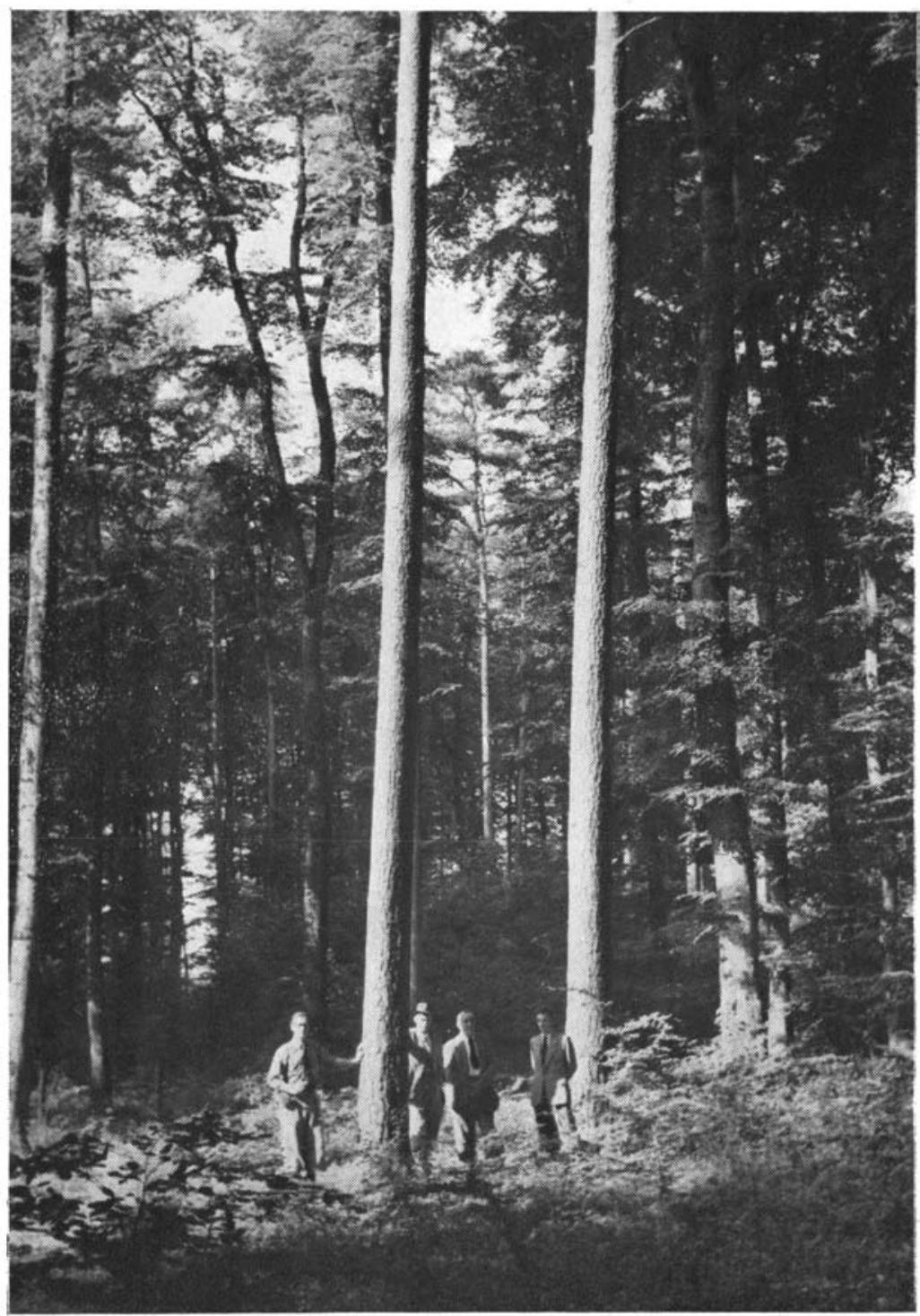

Bouquet de régénération: feuillus avec maintien de pins sylvestres de belle race pour la prociuction de gros bois de déroulage. Forêt de la Ville de Schaffhouse. Cl. Silvy I95I. 


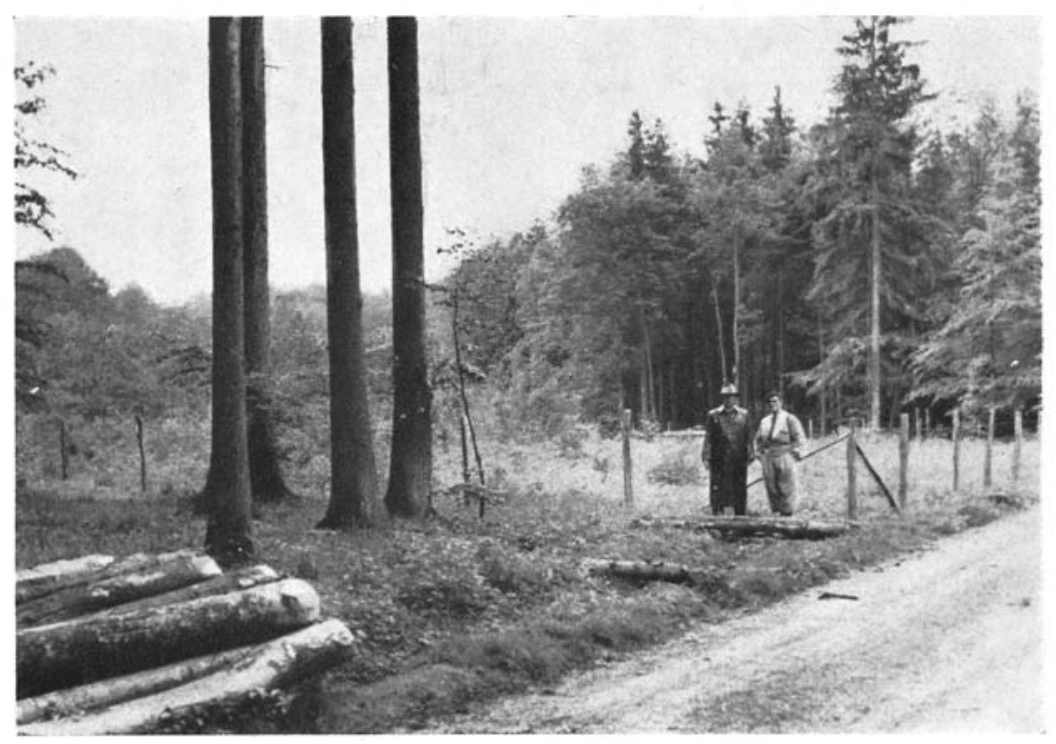

Bouquets d'épicéas et d'essences feuillues.

Canton du Ried. - Forêt d'enseignement de l'Ecole Polytechnique fédérale de Zurich.

Cl. Silvy i95I.

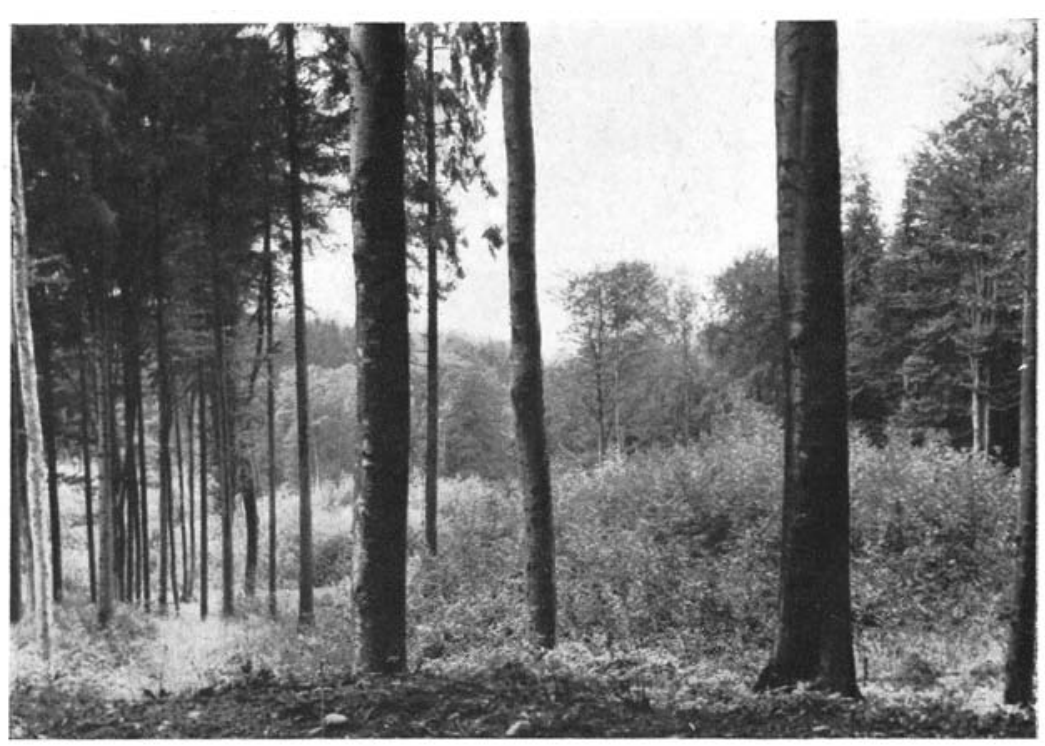

Bouquets de hêtres, sycomores et frênes, dans un ancien peuplement régulier d'épicéas avec groupes de vieux hêtres.

Canton d'Uetliberg. - Forêt d'enseignement de l'Ecole Polytechnique fédérale de Zurich. Cl. Silvy i952. 
moins approximatif qui conviendrait aux exploitations de peuplements, ni l'état vraiment inéquienne d'âges parfaitement mélangés, convenant aux exploitations d'arbres... Il n'est pas toujours facile de faire un choix entre ces deux formes (futaie pleine et futaie jardinée) dont les zones d'application se confondent et se pénètrent souvent en montagne, à cause des variations rapides d'un point à un autre de la forme du terrain, du climat local et des essences $)(5)$.

Il y a bien place, même pour les peuplements naturels, pour une forme intermédiaire. Mais on peut hésiter à rendre permanente, entre les éléments du peuplement, une différenciation qui peut exister à l'origine et s'atténuer jusqu'à disparaître par la suite.

Les méthodes d'aménagement assouplies permettent, notamment, dans le cas des sapinières, de pallier ces divers inconvénients.

Le pas a cependant été franchi et, depuis I95I, la littérature forestière s'est enrichie de nombreux articles consacrés au traitement du chêne en futaie par bouquets (6). Sans vouloir reprendre pour les analyser ces publications, nous pouvons cependant nous demander si leurs auteurs ne restreignent pas le sens de cette expression.

Le fait que cette méthode ait été proposée pour faciliter la culture du chêne dans l'Est de la France en remplacement de la futaie claire, ne doit pas limiter son application qui est plus générale.

Certes, son premier but restera pour nous de faire apparaitre la régénération naturelle. mais sous une forme morcelée. Mais, parce que le taillis-sous-futaie à convertir était riche en chêne, ne doit-on faire que des bouquets de chêne ? Parce que la futaie à irrégulariser était pure, faut-il consentir une monoculture par bouquets ?

Ce serait manifester comme un regret de ne pas pouvoir créer des futaies pleines et uniformes de ces essences, alors que la futaie par bouquets offre d'autres perspectives. Ce serait retomber dans ce " goît de l'homogénéité qui, dit GAYER, a étouffé ou du moins affaibli le sens de l'adaptation de la végétation forestière aux diverses stations; on ne sait plus localiser la culture en suivant partout les indications de la nature et en satisfaisant aux exigences spéciales à chaque station ») (3, p. 235).

$\mathrm{Ce}$ serait perpétuer des formations que $\mathrm{Ph}$. GuinIER reconnait assez éloignées du climax pour ne subsister que grâce à des interventions énergiques.

Ce serait faire fi d'un cri d'alarme lancé par $\mathrm{Ph}$. Duchaufour durant cette semaine d'information au cours de sa conférence sur la formation de l'humus forestier:

" La dégradation de la chênaie atlantique n'est pas imputable au chêne lui-même, mais à la sylviculture du chêne plus acidifiante parce qu'elle découvre fréquemment le sol ) (7).

Enfin, ce serait tourner le dos à des possibilités nouvelles offertes au propriétaire pour s'évader d'une sylviculture, peut-être valable pour les forêts de l'Etat, mais qui ne convient pas aux communes 
ou aux particuliers. Ce serait renoncer au programme que nous avons esquissé l'an dernier, ici même, à propos de la " Forêt productive ») en réclamant la variété des productions et des produits (8).

C'est en Suisse que nous irons chercher un exemple de futaie par bouquets qui facilitera notre exposé et notre discussion.

Depuis la rédaction de cette conférence, a paru un article très complet sur les tendances actuelles de la sylviculture en Suisse. Nous y renvoyons les lecteurs. P. Fourchy: «En Suisse. - Quelques aspects de la sylviculture contemporaine 》. Rev. forest. franc., mai I952, p. 315.

Nos collègues cnt bénéficié de conditions favorables pour préciser cette méthode qui est venue à point pour concilier les tendances des partisans et des adversaires d'un jardinage généralisé.

Sur un territoire à relief accentué, les variations de sol et d'exposition déterminent une extrême diversité dans les conditions écologiques sur des surfaces même restreintes. Les pédologues et phytosociologues suisses ont déterminé les associations climaciques correspondant à chacune des stations élémentaires. Les sylviculteurs en ont déduit les types de peuplement à cultiver et les traitements à appliquer.

Soulignons que ces types de peuplements ne représentent que rarement l'association végétale naturelle. Le climax ne constituant pas toujours la formation la plus avantageuse, le forestier cherche seulement à s'en rapprocher en choisissant, parmi les stades d'évolution, celui dont la composition et la structure répondent le mieux au but économique fixé. Il s'appuie pour cela sur un certain nombre des éléments de l'association naturelle, mais en y associant d'autres essences dont le tempérament s'accorde avec les conditions de station.

Le plateau suisse portait autrefois des peuplements feuillus d'essences variées, d'abord traités en taillis-sous-futaie. Les conversions directes par substitution d'essence en avaient transformé une partie en pessières pures dont les inconvénients étaient visibles.

L'application des principes du " Schweizerische Femelschlagbetrieb ) poursuivie depuis une trentaine d'années par ScHadeLIN et ses disciples, notamment LeIBUNDGUT, a permis de réaliser des futaies par bouquets non seulement dans les anciennes pessières pures et équiennes, mais dans les restes des anciens taillis-sous-futaie, dont la conversion s'est trouvée réalisée rapidement et avantageusement.

Ces principes sont les suivants:

I ${ }^{\circ}$ La création par bouquets, suivant la méthode préconisée par GAYER, est réalisée:

a) en amorçant la régénération naturelle par ouverture des trouées jardinatoires,

b) en introduisant des groupes d'essences améliorantes dans les 
peuplements purs résineux ou d'essences à croissance rapide dans les taillis.

$2^{\circ}$ Le choix et la localisation des essences sont déterminés conformément aux données de la pédologie et de la phytosociologie.

$3^{\circ}$ La disposition des bouquets sur le terrain s'inspire des possibilités de débardage (respect de l'ordre spatial).

$4^{\circ}$ Les bouquets une fois créés, naturellement ou artificiellement, sont traités comme des éléments distincts et évoluent indépendamment les uns des autres, suivant la nature et le tempérament des essences qui les constituent et suivant lav qualité des sols qui les portent.

Nous nous rendons compte de ce que l'exposé de ces principes peut avoir d'artificiel et de dogmatique. Pour dissiper une telle impression qui est contraire à tout ce que les sylvicultẹurs suisses ont voulu faire de cette technique qui leur est propre, nous essaierons de donner une image concrète de ce que serait une futaie par bouquets.

Nous prendrons un exemple précis dans une forêt des environs de Nancy où, sur marnes du Lias, a été entreprise depuis I869, à partir d'un taillis-sous-futaie, ce que nous appelons une " conversion en chêne ).

Les deux schémas réunis sur la figure ci-contre, tendent à représenter une conversion de taillis-sous-futaie telle qu'elle aurait pu être réalisée en France par la méthode classique (schéma A) et telle que la concevraient les Suisses par la méthode (" affinée ) de futaie par bouquets (schéma B).

L'interprétation du relief est possible grâce à quelques lignes figuratives qui ne représentent cependant pas des limites parcellaires. Les grandes parcelles françaises vont de la route de faite au fond du vallon; elles représentent une surface moyenne de I 3 ha. Les bouquets suisses ne sont délimités que pour les résineux. Il est loisible d'imaginer dans chaque type d'association des bouquets feuillus correspondant aux groupes, d'essences ou d'âges divers, maintenus ou transformés suivant les indications culturales et économiques.

Dans la pratique, ces bouquets seraient délimités d'après les variations observées dans les peuplements existants et qui sont révélés par la photographie aérienne. Ils sont alors superposés aux hachures caractéristiques des types d'associations végétales naturelles qui servent de base à l'établissement du plan de traitement.

Les types d'associations que nous avons adoptés sont ceux décrits par divers auteurs pour le Nord-Est, de la France et qui semblent se rapprocher le plus des types définis par KосH et ETTER pour le plateau suisse, bien qu'en raison des différences de climat, les caractéristiques ne soient pas toutes concordantes. 


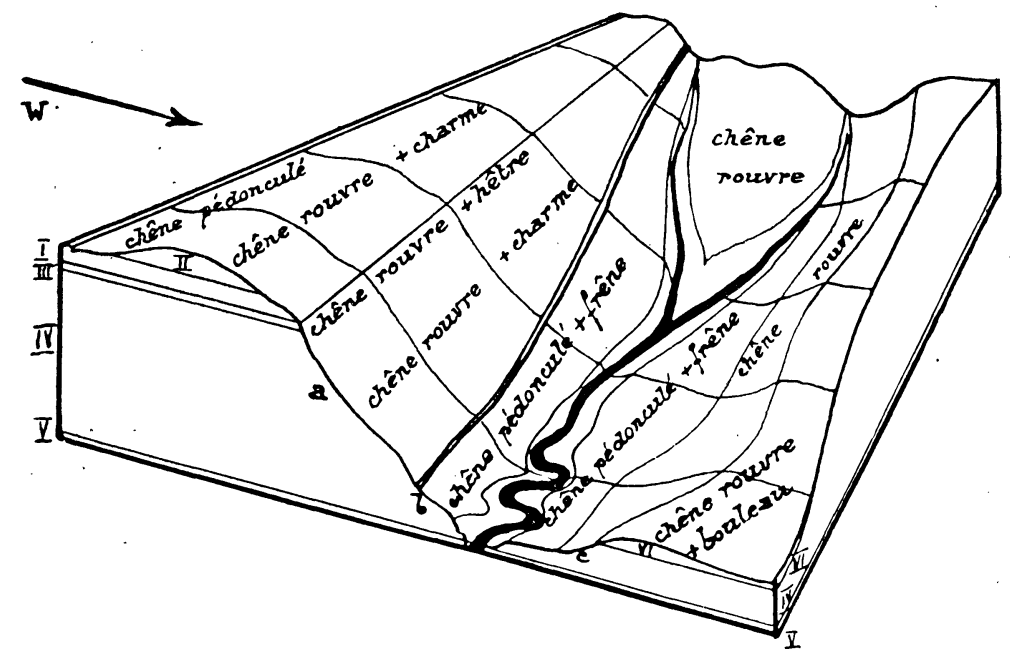

Schéma A.

Ancien taillis-sous-futaie traité suivant la méthode française de conversion.

Niveaux géologiques

I Marnes toarciennes.

$$
\mathrm{pH}=5,5
$$

II : Placage de limons des terrasses.

$$
\mathrm{pH}=5
$$

III Grès médioliasiques.

$$
\mathrm{pH}=6
$$

$$
\text { IV charmou- } \begin{gathered}
\text { Marnes } \\
\text { thiennes }
\end{gathered}\left\{\begin{array}{cc}
\text { a) Versant Est. } & \text { Concurrence du charme. } \\
\mathrm{pH}=5,5 & \text { Concurrence du charme. } \\
\mathrm{pH}=6 & \\
\text { c) Versants Sud et Ouest. Disparition du charme. Entrainement } \\
\mathrm{pH}=5 & \begin{array}{l}
\text { et appauvrissement des horizons su- } \\
\text { perficiels. Faible valeur du chêne. }
\end{array}
\end{array}\right.
$$

V Calcaire ocreux.

$$
\mathrm{pH}=6,5 \grave{\mathrm{a}} 7
$$

Points menacés ou défectueux

Remontée du plan d'eau - Horizon à gley - Molinie - Difficultés de régénération.

Danger d'acidification par découvert.

Concurrence du hêtre.

Absence de sous-étage, découvert accentué du sol, développement relatif des morts-bois.

VI Placage d'alluvions anciennes caillou-Acidification par découvert. Peupleteuses. ment clair. Faible valeur du chêne. $\mathrm{pH}=4,5$ 


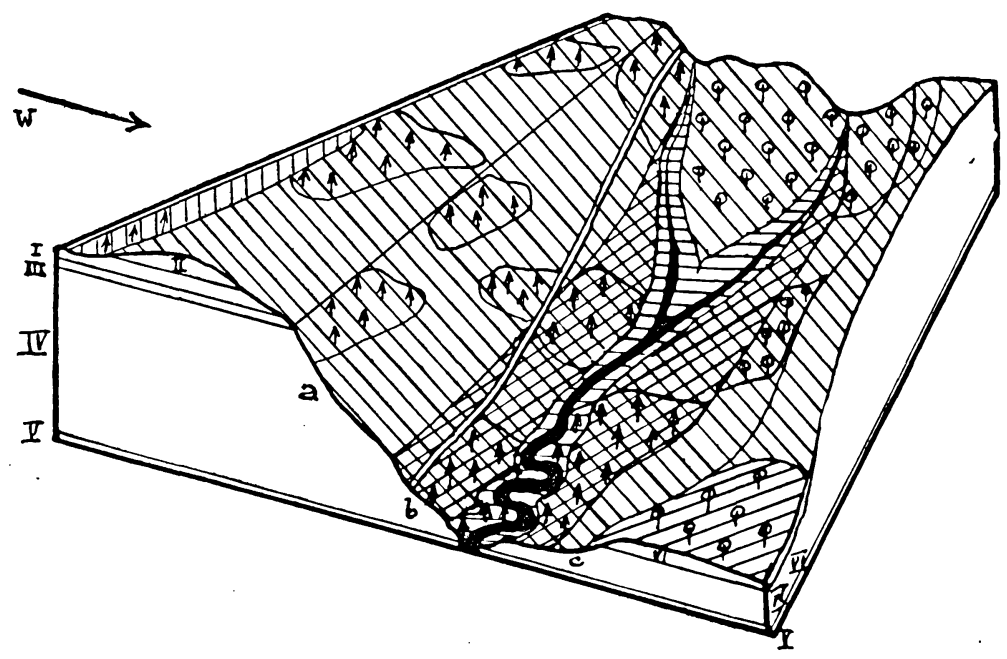

Schéma B.

Ancien taillis-sous-futaie traité suivant la méthode suisse de Futaie par bouquets.

Types d'associations végétales naturelles

I Querceto-carpinetum medioeuropaeum molinietosum

II Querceto-carpinetum luzuletosum

III

IV

IV Querceto-carpinetum aretosum

IV Querceto-carpinetum luzuletosum

V Cariceto remotae Fraxinetum

VI Querceto-betuletum
Indications culturales et économiques

Limiter l'étendue des coupes. Maintenir ou rétablir le mélange. Bouquets feuillus d'aune glutineux ou de chêne rouge. Enrichissement à i'aide de thuya géant et épicéa.

Intérêt économique du chêne rouvre à maintenir dominant avec charme et tilleul. Bouquets de mélèze d'origine choisie.

Bouquets de hêtre et de sapin. Chêne rouvre à maintenir à l'état d'individus isolés au milieu de groupes de feuillus et de résineux.

Intérêt des chênes en peuplements mélangés: charme, hêtre, sapin. Enrichissement en Douglas.

Intérêt des chênes en peuplements mélangés: merisier, orme, tilleul, sycomore, charme. Enrichissement bn Abies grandis et épicéa.

Faible valeur du chêne. Bouquets d'essences mélangées: tilleul, fruitiers, chêne associé. Enrichissement en pin sylvestre avec sous-étage feuillu.

Culture d'essences à croissance rapide en peuplements mélangés. Bouquets d'aune glutineux, frêne, orme, enrichis par pin Weymouth, thuya géant et $A$ bies grandis.

Médiocre valeur technologique du chêne. Amélioration par résineux et feuillus sous-introduits. Bouquets mélangés de pin sylvestre, chêne rouge, et aune blanc dans les clairières peu enherbées. 
L'examen de la conversion réalisée par la méthode française fait apparaitre certaines défectuosités.

Bien que l'ensemble forme une futaie régulière conforme au but fixé par l'aménagement, il reste des problèmes non résolus : la rẻgénération est incomplète dans certains cantons où la remontée du plan d'eau et l'envahissement par la molinie compromettent l'avenir; ailleurs l'absence de sous-étage met en cause le maintien du sol en bon état; ou encore la coexistence du chêne rouvre et du hêtre au niveau où affleurent les grès médio-liasiques rend délicate la conduite du peuplement si l'on veut maintenir le chêne dominant; enfin, la régénération de l'essence " précieuse » reste aléatoire dans certaines parcelles déjà envahies par des semis naturels de charme et de frêne, sous un couvert qui exclut.le chêne pédonculé, mais qui ne peut être réduit brusquement sans risque d'envahissement par les essences secondaires et les morts-bois.

Les dangers et les inconvénients que nous signalons se trouvent réduits si l'on abandonne la recherche de parcelles homogènes pour créer des bouquets où les tendances naturelles des divers éléments seront utilisées, voire même favorisées.

Les considérations économiques et sylvicoles conduisent à ne maintenir le chêne dominant que dans les stations où il est susceptible d'atteindre de fortes dimensions et de fournir des bois de valeur. Ailleurs, il cédera le pas jusqu'à n'être plus qu'associé aux essences secondaires ou introduites qui fournissent des produits plus rémunérateurs.

Ne nous méprenions pas sur le véritable sens de la futaie par bouquets. Vouloir la limiter à une technique sylvicole propre à assurer ou faciliter une régénération serait trahir la pensée de ceux qui l'ont mise au point.

Mais avant d'examiner les cas où serait justifiée son application, mesurons le bouleversement qu'elle apporterait dans notre conception française de la forêt aménagée. Gurnaud passa pour révolutionnaire lorsqu'il parla de futaie jardinée à ses contemporains. La théorie du jardinage et la méthode du contrôle devaient cependant permettre d'ordonner dans le temps une irrégularité qui ne vise que les âges d'un petit nombre d'essences.

La considération des schémas précédents peut faire naitre de nombreuses objections: de la part des aménagistes qui ne voient pas comment concilier cette méthode avec leur sens de l'ordre et des prévisions dans les exploitations; de la part des économistes qui objectent que la. culture d'essences dites secondaires n'est pas rentable dans les conditions actuelles du marché; de la part des gestionnaires qui voient des difficultés d'application avec un personnel trop peu nombreux et en raison des modes de vente jusqu'ici utilisés.

Nous reprenons chacune de ces objections: 
$\mathrm{I}^{\circ}$ du point de vue des aménagements:

Il est vrai que la futaie par bouquets telle qu'elle est pratiquée en Suisse est " affranchie de tout dogme et de toute entrave " (9) résultant, soit d'un terme d'exploitabilité pour les essences, soit d'une date de réalisation pour les bouquets, soit d'une possibilité annuelle impérative.

Nous voici très loin des aménagements rituellement appliqués en France. où se trouvent successivement déterminés un terme d'exploitabrilité ou une révolution, une possibilité annuelle, et un règlement d'exploitation.

Nous nous trouvons en France tenus à plus de rigueur, en raisọn de l'insuffisance numérique et des fluctuations du personnel de gestion. L'aménagement est un guide utile pour renseigner les agents d'exécution successifs et assurer la continuité des opérations.

Mais nous ne devons pas nous méprendre sur la valeur réelle des notions précédentes notamment en période de conversion ou de transformation. A quoi correspond un terme d'exploitabilité lorsque la coupe porte sur des réserves de taillis-sous-futaie dont les âges varient de $n$ révolutions ? A-t-on une telle certitude en appliquant une possibilité par volume calculée à partir d'un taux d'accroissement unique pour les arbres à exploiter, alors qu'ils manifestent des croissances très variables ? L'état normal sera-t-il réalisé par l'application des prescriptions d'un aménagement alors que cet état normal n'a pas été défini ?

N'est-ce pas l'occasion de rappeler cette phrase de Biolley: « Il n'y a pas d'autre état normal que celui qui assure l'utilisation intégrale de toutes les sources de la production )! (Io).

Ce principe trouve une application particulièrement exacte dans la futaie par bouquets où le choix des essences, lié à la considération de station. fixe la capacité de production en bois. Rien ne s'oppose d'ailleurs à ce que le forestier, par des relevés et des mesures d'accroissement, fixe plus précisément cette capacité et arrive ainsi à différencier l'avenir de chacun des bouquets.

Plus grave semble être l'abandon de la notion de parcelle unité culturale, généralement admise dans les traitements réguliers. Cette notion commode admet que, sur toute la surface de la parcelle, le peuplement est suffisamment homogène pour être traité de la même manière. Avec les bouquețs, d'âges variés, d'essences diverses, exploités à des termes différents, la parcelle subsiste seulement sous forme de limites de gestion. Les plans de forêts se compliquent et doivent être complétés par des calepins de terrain ou des fiches parcellaires où se trouvent figurés les bouquets et leur évolution présumée. (Nous renvoyons, pour plus amples détails sur cette cartographie, à une analyse d'un article de H. LeibundGut (I I) qui figure dans la Revue des Revues de ce numéro.)

La notion de parcelle unité culturale peut se légitimer dans les forêts de plaine, où de vastes étendues de sols de même origine et 
au même stade d'évolution, peuvent porter des peuplements homogènes. Nul ne songe à introduire la futaie par bouquets dans les chênaies à charme du Centre et de l'Ouest, sur sols d'argile à silex. Les résultats des régénérations réussies apparaissent dans les surfaces étendues, couvertes de peuplements de classes d'âges bien réparties.

Si parfois, une variation locale de station apparait, la structure du peuplement (ou sa composition) la reflète; le forestier a peutêtre, en ce point, dû faire appel à quelques artifices (drainages, crochetages, regarnis) pour maintenir l'unité, et dans l'application de sa doctrine, et dans la physionomie de sa forêt.

Encore admissible dans certaines sapinières de moyenne altitude, sur versants continus à faibles variations de sol et d'exposition, la notion de grandes parcelles homogènes ne se justifie plus lorsque les conditions de station varient constamment d'un point à un autre et ne permettent pas l'application d'un traitement uniforme. Notre but n'est pas de discuter des aménagements de futaie par bouquets, mais seulement d'attirer l'attention des auditeurs sur les répercussions que peut avoir une technique sylvicole nouvelle sur des doctrines d'aménagement considérées comme classiques. Nous souhaitons en même temps que soient étudiées des formules nouvelles suffisamment souples pour que les opérations culturales puissent être menées au mieux des transformations envisagées.

\section{$2^{\circ} d u$ point de vue de l'économie:}

Prôner la culture d'essences secondaires comme le tilleul, le charme, peut sembler paradoxal à une époque où la faveur du marché va à certaines catégories de bois durs et aux essences résineuses.

Dans l'exposé fait au cours de la Semaine d'Information de I95I (8), nous insistions sur. les caractères de la " forêt productive » et notamment sur la nécessité d'offrir à l'utilisateur futur la plus grande variété possible de produits. Une essence, recherchée aujourd'hui, peut être télaissée demain. La forêt mélangée, offrant de plus nombreux débouchés, diminue les risques d'un marché des bois en rapide évolution.

Nous avons la chance de disposer d'un patrimoine forestier particulièrement riche en espèces, en raison de la variété des stations, mais grâce auısi, il faut le reconnaître, à l'exploitation en taillis et taillis-sous-futaie des siècles précédents. Dans ces peuplements, ont pris place, à la faveur d'apports successifs, de nombreuses essences dites " secondaires " mais dont la valeur doit être soulignée. La lecture des statistiques résultant des grandes ventes permet de suivre les fluctuations des prix des 7 ou 8 grandes essences, mais ne rend pas compte de l'intérêt des autres. La pratique des ventes faites en bloc et sur pied et par parcelles entières, ne permet pası la distinction entre essences diverses. Il faut se reporter aux cours indicatifs fournis par diverses revues techniques, pour voir que certaines de ces 
essences atteignent ou dépassent la valeur des hêtres et des chênes.

Les chiffres qu'on y trouve ne tiennent pas compte des plus-values résultant de longueurs exceptionnelles, qualités technologiques particulièrcs, etc... qui permettent à des bouleaux de déroulage (I30, I $40 \mathrm{~cm}$ de circonférence) d'être achetés dans l'Est de la France par des importateurs stisses à 8.000 francs le mètre cube sur wagon, et à des grumes d'érable et de fruitiers d'atteindre ro.ooo francs le mètre cube.

Ces chiffres réunis en avril 1952 ont aujourd'hui perdu toute valeur absolue. Ils permettent de discuter la valeur \& relative 》 des essences dites secondaires.

Le maintien de ces essences secondaires encore visibles dans les taillis-sous-futaie en conversion est désirable au point de vue cultural comme au point de vue économique.

La futaie par bouquets concilie la production des essences principales avec une récolte limitée de bois d'essences disséminées que risque de faire disparaitre la régénération parr parcelles entières traitées de manière uniforme. Et la pratique des exploitations en régie achèvera cette revalorisation en permettant de distinguer et d'organiser un véritable marché de ces bois.

\section{$3^{\circ} d u$ point de vue de la gestion:}

Nous touchons là au point critique: futaie par bouquets implique sylviculture intensive, c'est-à-dire personnel nombreux et faibles surfaces à gérer.

" La forme de ce peuplement rend plus nécessaire encore une étude attentive de ses diverses parties et des forces de production disponibles; on ne saurait voir là une voie toute tracée, chaque mesure doit au contraire y être dictée par les circonstances locales et prise en vue de chaque cas spécial. De plus, le soin du peuplement y absorbe une bonne partie de l'activité du préposé, comme dans tous les systèmes qui se caractérisent par de grandes différences d'âges, et où l'on a en vue le dégagement de certains sujets destinés à donner des bois d'œuvre. Cette nécessité de culture individuelle devient dans les peuplements irréguliers et mélangés une tâche souvent difficile, qui demande d'autant plus.d'activité et de discernement que les surfaces sur lesquelles on opère sont plus constamment disséminées. Il s'ensuit que ces traitements ne peuvent s'appliquer que dans des cantonnements d'étendue moyenne ) (3, p. I78).

A supposer même que le problème ait reçu une solution, il faut encore mesurer à quel point cette forme complexe de peuplement s'éloigne de la conception française de la forêt aménagée. Tous les efforts depuis $5_{50}$ ans ont tendu à rendre simple l'image des divers peuplements. Ce souci d'ordre et de clarté avait abouti aux aménagements par affectations. D'un coup; on envisage d'abandonner ce qui passait pour un progrès. Les agents d'exécution, insuffisamment stables, pourront-ils se familiariser avec une telle forme et 
( voir )) le bouquet de résineux à dégager au milieu des feuillus de la parcelle I4, aussi bien qu'un peuplement homogène " sortant de $3^{\text {e }}$ affectation $)$, par exemple?

Mais combien trouve-t-on de forêts dont les peuplement soient parfaitement homogènes sur toute l'étendue d'une parcelle ? L'exemple pris plus haut montre que, sur une surface même réduite, apparaissent, suivant les niveaux, le chêne pédonculé, le chêne rouvre, le hêtre, le frêne, etc... justiciables chacun d'un traitement approprié.

L'exécutant, muni de calepins tenus à jour, ne sera plus désorienté s'il prend l'habitude de lier les bouquets aux variations de terrain et d'exposition, et de réaliser visuellement ce que prévoit l'aménagement.

La question des ventes de coupes se pose enfin. Les acquéreurs ne seront-ils pas rebutés par des difficultés accrues d'exploitation et de débardage ? La variété de produits ne rendra-t-elle pas plus difficile le placement de coupes éparses au milieu de bouquets, alors que la faveur va aux lots provenant d'exploitations concentrées et fournissant des produits homogènes ?

Là encore. nous rappellerons que les futaies sur souches sont formées d'éléments disparates et que les produits récoltés sont fort peu homogènes. L'homogénéité se trouvera peut-être mieux réalisée à l'intérieur d'un bouquet.

Mais il reste la dispersion des produits qui est réelle, et le débardage plus difficile qui ne peut être réalisé qu'en étudiant au préalable une disposition des bouquets liée aux voies de vidange.

Sur ces point encore, la régie directe atténue les inconvénients signalés.

Cette discussion des bouleversements qu'apporterait à nos habitudes la technique de la futaie par bouquets nous amène à conclure sur les possibilités d'application de cette méthode.

Nous estimons que le temps n'est pas encore venu pour les Français, de généraliser l'emploi d'une sylviculture aussi intensive; les possibilités en personnel, l'organisation administrative et les conditions économiques actuelles ne s'y prêtent pas.

Il ne peut être question de modifier les méthodes qui ont fait leur preuve dans les grands massifs du Centre et de l'Ouest, où les conditions naturelles sont particulièrement uniformes. L'organisation de la production à l'échelon national doit laisser à ces forêts leur spécialisation, qui est de fournir des bois de chêne de fortes dimensions et de grande valeur qui ne peuvent être réalisés ailleurs.

Les sapinières vosgiennes ou jurassiennes, une fois restaurée leur ambiance par un mélange raisonnable de feuillus, peuvent continuer à être traitées en grands massifs. De même, certaines forêts domaniales, devenues des hêtraies, sous réserve d'y rendre aux 
essences secondaires une place limitée, sont justiciables de ces traitements uniformes.

Par contre, dès que les conditions naturelles, les dimensions du massif, les nécessités de transformation, ou la nature du propriétaire, s'opposent à la réalisation d'une futaie pleine présentant une suite schématique de peuplements, ou ne permettent d'escompter qu'un faible taux de placement, il est indispensable de rechercher une forme de traitement plus souple et plus économique. Sur les 3.500.000 hectares de taillis-sous-futaie accusés par les statistiques, il y a bien place pour toute méthode susceptible d'accélérer leur transformation.

Nous considérons que, dans les forêts soumises, elle est tout indiquée pour prendre en main les queues d'affectations, les portions de forêts où la conversion se révèle incertaine.

Les méthodes classiques conduisent bien souvent à une impasse; à vouloir mieux prépurer les peuplements en prolongeant la période d'attente, on s'expose à un vieillissement généralisé de l'ensemble des éléments, dont les derniers ne viendront en tour de régénération qu'à une époque où les semenciers seront surannés et la qualité des bois rendue douteuse.

Il ne s'agit pas de détruire, ni même de bouleverser la structure intime de nos peuplements qui, dans l'ensemble, ne sont pas trop éloignés de l'association naturelle, ainsi que le fait ressortir le schéma A. Mais il convient de corriger ce qui apparaît défectueux pour la stabilité ou pour la productivité de la forêt.

Les directives seront donc les suivantes:

$\mathrm{I}^{\circ}$ établir un plan d'amélioration proportionné aux moyens dont on dispose, notamment en personnel,

$2^{\circ}$ s'appuyer sur ce qui existe,

$3^{\circ}$ se rapprocher du " groupement écologique ", sans rechercher trop systématiquement des peuplements homogènes en essences dites " nobles ",

$4^{\circ}$ associer les essences dites secondaires en tenant compte des conditions locales de station et de marché,

$5^{\circ}$ introduire dans les vides et les portions dégradées des essences améliorantes et des essences à croissance rapide susceptibles de compenser les sacrifices consentis pour l'amélioration des peuplements existants,

$6^{\circ}$ retourner à un cadre suffisamment simple et schématique dans les grands massifs soumis au régime forestier,

- conserver plus de souplesse dans les petits massifs et dans les forêts particulières.

Nous ne prétendons pas inventer une technique déjà mise au point par de nombreux forestiers aussi bien en forêt privée qu'en domaine soumis. Les exposés faits par P. Hirsch au Comité des Forêts (12) reflètent imparfaitement tout le travail accompli et mené à bien par ce protagoniste du chêne dans les forêts d'Amboise et de 
Conches, par exemple. L'article de H. de Metz-Noblat (I3) et la forêt qu'il a reconstituée sont des preuves du soin avec lequel certains propriétaires travaillent leurs peuplements et cherchent à tirer parti de tous les éléments disponibles. Tout ce qu'ils ont exposé est marqué du sceau du praticien.

Si nous avons voulu faire de la futaie par bouquets un exposé plus théorique, plus schématique, c'est d'abord parce qu'il faut bien, de temps à autre, reprendre les notions de base et les rattacher ou les opposer les unes aux autres; c'est qu'ensuite il peut apparaitre diverses interprétations de la même idée, et la futaie par bouquets se prête à des formes si variées qu'il est nécessaire de les bien préciser ; c'est qu'enfin, on n'a pas toujours utilisé toutes les possibilités d'une telle technique.

Nous la recommandans aux propriétaires parce qu'ils y trouveront, comme disait $\mathrm{H}$. de Metz-Noblat, la forme la mieux appropriée à leurs moyens et à leurs besoins.

En terminant cet exposé, nous n'aurions pas atteint notre but si nous laissions à nos auditeurs l'impression que la futaie par bouquets représente un but en soi. Nous la considérons seulement comme un moyen, peut-être transitoire, parfois durable, de rendre une forêt plus proche de l'idéal que recommande la science et que réclame l'économie moderne.

Cette méthode, dans sa forme affinée, nous est révélée par les réalisations longuement mûries des sylviculteurs suisses. Les propriétaires français ont trop souvent reproché aux forestiers d'Etat de mettre au point et en pratique des méthodes qui ne valent que pour les grandes forêts domaniales, pour ne pas saisir l'occasion qui s'offre aujourd'hui. Il appartient aux Ingénieurs des Eaux et Forêts de prendre la tête du mouvement dans l'application d'une méthode qui a besoin d'être précisée sur le terrain. Qu'ils recherchent dans leur service quelques cantons bien choisis susceptibles de devenir de véritables forêts de démonstration pour une région donnée. Et mieux, qu'ils aident les particuliers à réaliser dans leurs propres forêts cette économie des petits massifs qui concilie tant de points de vue et rétablit pour le gibier des conditions idéales.

Aux grandes forêts sans mystère, tracées comme les perspectives de LE Nôtre, beaucoup doivent aujourd'hui préférer le boisé plus secret où, comme en un jardin, se trouvent encloses toutes les variétés de production que la nature permet.

P. Silvy-Leligois.

\section{REFERENCES BIBLIOGRAPHIQUES}

I. Ch. Broilliard. - Le traitement des bois en France. Préface de la $2^{\circ}$ édition. Ed. Berger-Levrault, Paris-Nancy, I894.

2. G. Bagnéris. - Manuel de sylviculture. Ed. Berger-Levrault, ParisNancy, $I^{\text {re }}$ édition, I873; $2^{\circ}$ édition, I878. 
3. K. Gayer. - Traité de Sylviculture (Traduction E. Visart de Bocarmé). Imprimerie G. Stock. Bruges, I90I.

4. G. TURNER. - Transformation des pessières en peuplements mélangés. Bull. Comité des Forêts, $\mathrm{n}^{\circ} 84$, I95I.

5. G. Hufrel. - Economie forestière, tome III, p. 403. Librairie Agricole de la Maison Rustique. Paris, I926.

6. F. de Lemps. - Futaie jardinée par bouquets et futaie de chêne par bouquets. Rev. forest. franc., septembre I95I, p. 577.

Desouche. - Coupes temporaires de futaies par bouquets. Rev. forest. franc., janvier I952, p. 4.

7. Ph. Duchaufour. - Dégradation des sols sur les versants chauds. Rev. forest. franç., février I95I, p. 107. Voir aussi p. I05, références aux articles de Noirfalize et Galoux.

8. P. Silvy-Leligois. - La forêt productive. Rev. forest. franç., janvier I 952 , p. 20.

9. H. LeibundGut. - Grundzüge der Schweizerichen Waldbaulehre. Schw.. Zeitschr. für Forstzeesen, nos 9-10, 1948.

io. H. Biolley. - Phrase extraite d'une lettre personnelle et citée par: L. Schaeffer. Gurnaud et le jardinage. Livret du Cinquantenaire de la Sté Forestière de Franche-Comté. Imprimerie Declenne. Lons-le-Saunier, 1947, p. 239.

i. H. Leibunngut. - Ueber die Planung von Bestandesumwandiungen. Schw. Zeitschr. für Forstwesen, $\mathrm{n}^{\circ \mathrm{s}}$ IO-I I, 1947.

I2. P. Hirsch. - Remarques à propos de la conversion en futaie de hêtre des taillis-sous-futaie sur plateaux calcaires du N.E. de la France. Bull. Comité des Forêts, $\mathrm{n}^{\circ}$ 82, I949, p. II89. Voir aussi compte rendu du voyage du Comité des Forêts en I950. Bull. Comité des Forêts, n 83, I950, p. I3I I.

13. H. de Metz-Noblat. - La reconstitution d'une forêt. Rev. forest. franc., avril 1950, p. 207.

\section{Manchettes bibliographiques}

Il a paru souhaitable de faciliter la manipulation, le dépouillement et le classemcnt des revues en les soumettant à une certaine unification de' présentation. Dans ce but, chaque fascicule porterait en un endroit bien apparent, les données bibliographiques essentiellcs, qui constituent 1 ' « état civil $\gg d u$ fascicule: c'est ce qu'on nomme la manchette bibliographique.

Une norme en préparation $Z$ 44-003 prévoit comme emplacement pour la manchette, le bas de la première page de couverture et de la première page de texte.

Le titre serait donné en abrégé. Il serait suivi d'indications sur la tomaison, le numéro du fascicule, la pagination des pages extrêmes, le lieu de publication, le millésime et la date de publication.

On note que pour éviter dans l'indication des mois toute confusion entre le romain II (deux) et l'arabe ir (onze), on pourrait abréger les noms des mois d'après leur forme latine, comme suit: jan., feb., mar., apr.. mai., jun., jul., aug., sep., oct., nov., dec.

Les noms des mois sont ainsi réduits à trois lettres seulement, sans confusion possible entre eux.

Le présent numéro de la Revue aurait ainsi pour manchette:

Rev. for. franç. $\mathrm{T} . \mathrm{V} \mathrm{n}{ }^{\circ} 3$ pp. 157-244 Nancy 1953 mar. 